
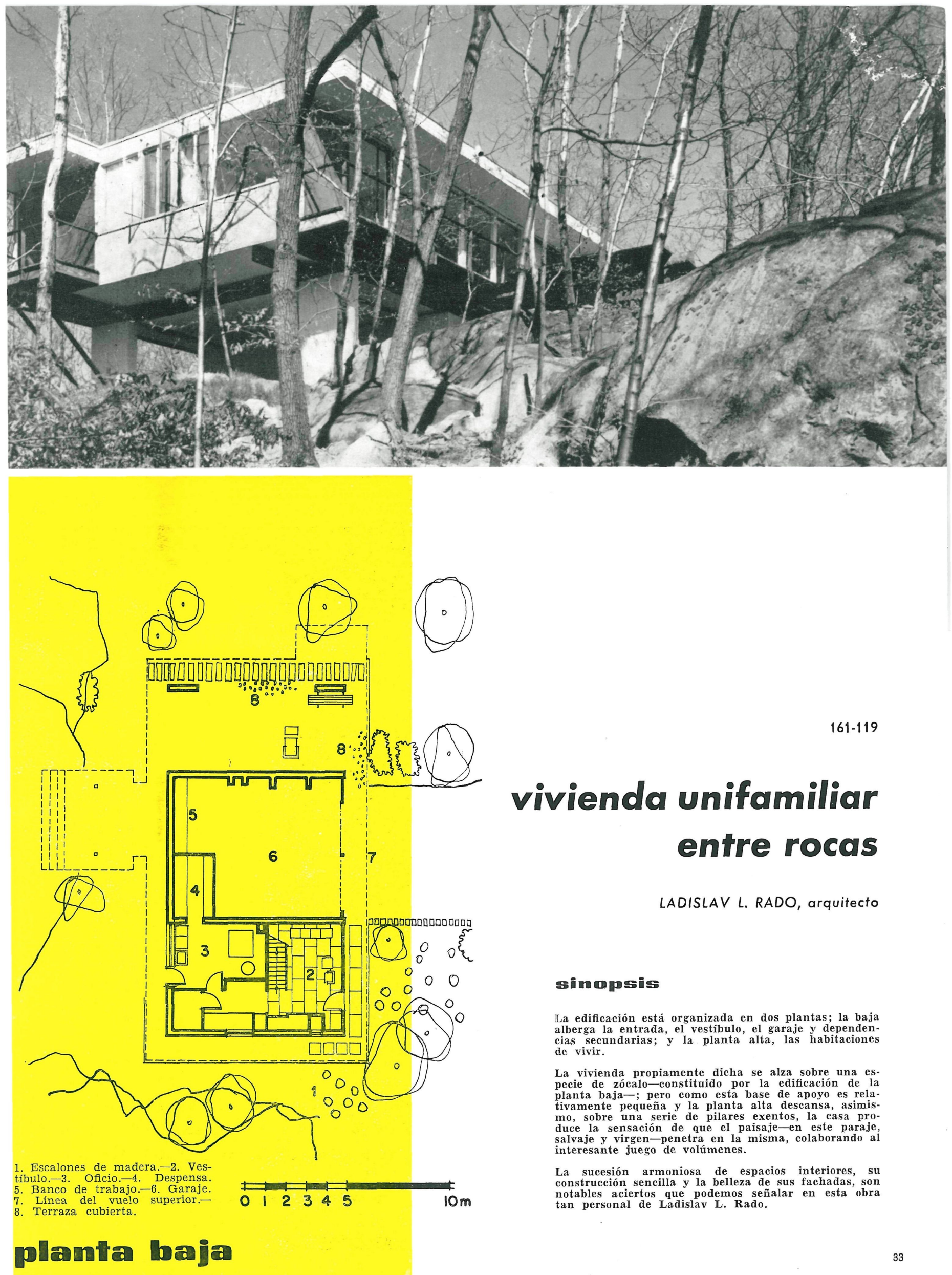

$161-119$

\title{
vivienda unifamiliar entre rocas
}

LADISLAV L. RADO, arquitecto

\section{simenpsis}

La edificación está organizada en dos plantas; la baja alberga la entrada, el vestíbulo, el garaje y dependencias secundarias; y la planta alta, las habitaciones de vivir.

La vivienda propiamente dicha se alza sobre una especie de zócalo-constituido por la edificación de la planta baja-; pero como esta base de apoyo es relativamente pequena y la planta alta descansa, asisdu, so sensacón de pul el paisajeinteresante juego de volumenes. pe volúmenes.

La sucesión armoniosa de espacios interiores, su construcción sencilla y la belleza de sus fachadas, son notables aciertos que podemos señalar en esta obra tan personal de Ladislav L. Rado. 


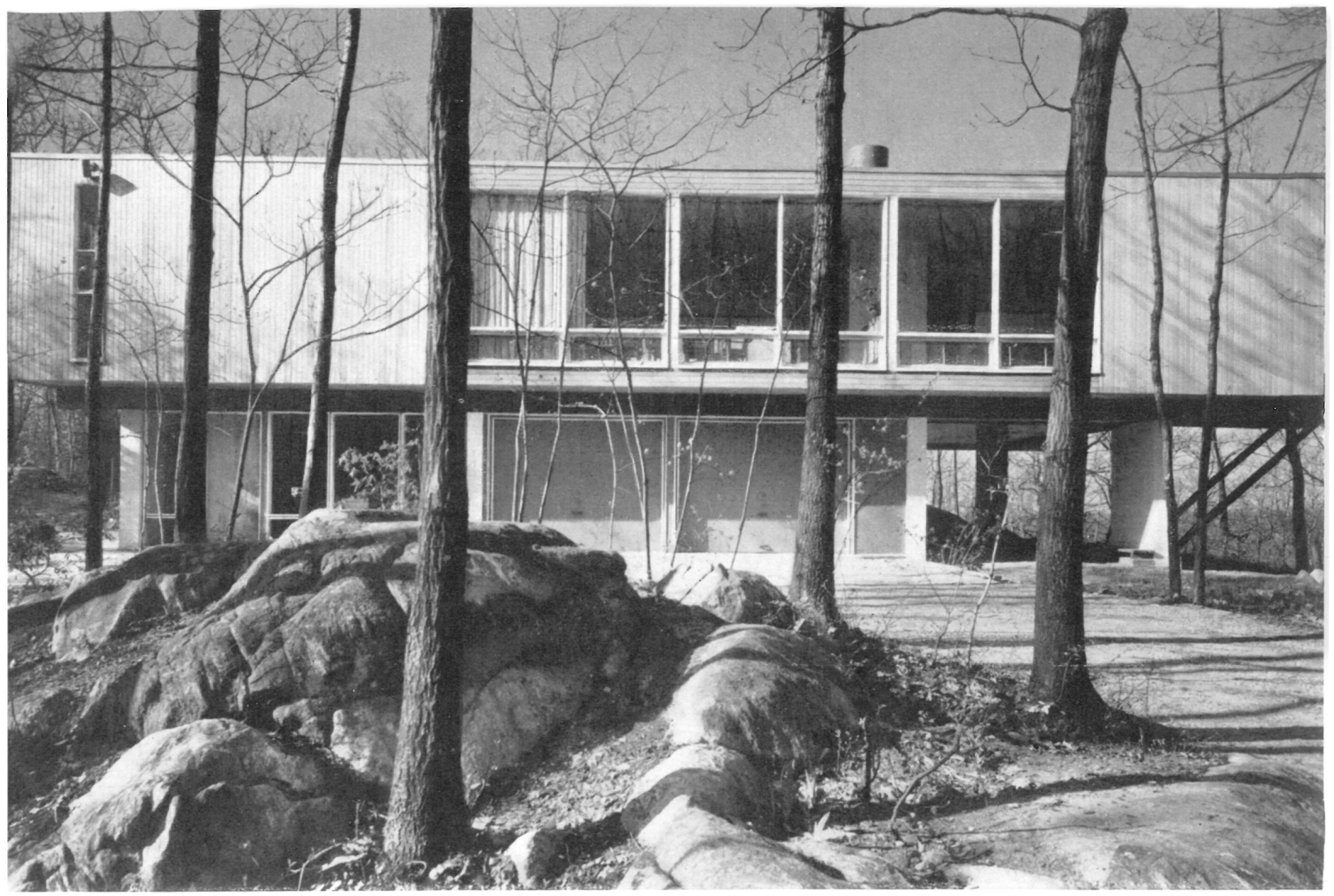

Dada la circunstancia de ser extraordinariamente rocoso el terreno en Shappaqua-un suburbio de Nueva York-, a nadie se le pasó por la imaginación edificar sobre él. Pero, como siempre ocurre, hubo una excepción: fue el arquitecto Rado, el cual, llevado precisamente por la belleza salvaje y virgen del lugar decidió construir allí su propia casa.

Es más: con objeto de respetar las características del emplazamiento, no cambió sensiblemente su topografía irregular. La edificación la organizó en dos plantas: en la baja instaló la entrada, el vestíbulo, el garaje y dependencias secundarias; y en la planta alta, las habitaciones de vivir -diurnas y nocturnas-.

La vivienda propiamente dicha se alza sobre una especie de zócalo-constituido por la edificación de la planta baja-, del que sobresale considerablemente por una serie de voladizos.

Como la base de apoyo construida es relativamente pequeña, y la planta alta descansa, asimismo, sobre una serie de pilares exentos, la casa suscita la sensación de que el paisaje penetra en la misma, ayudando con esto al propio movimiento de volúmenes.

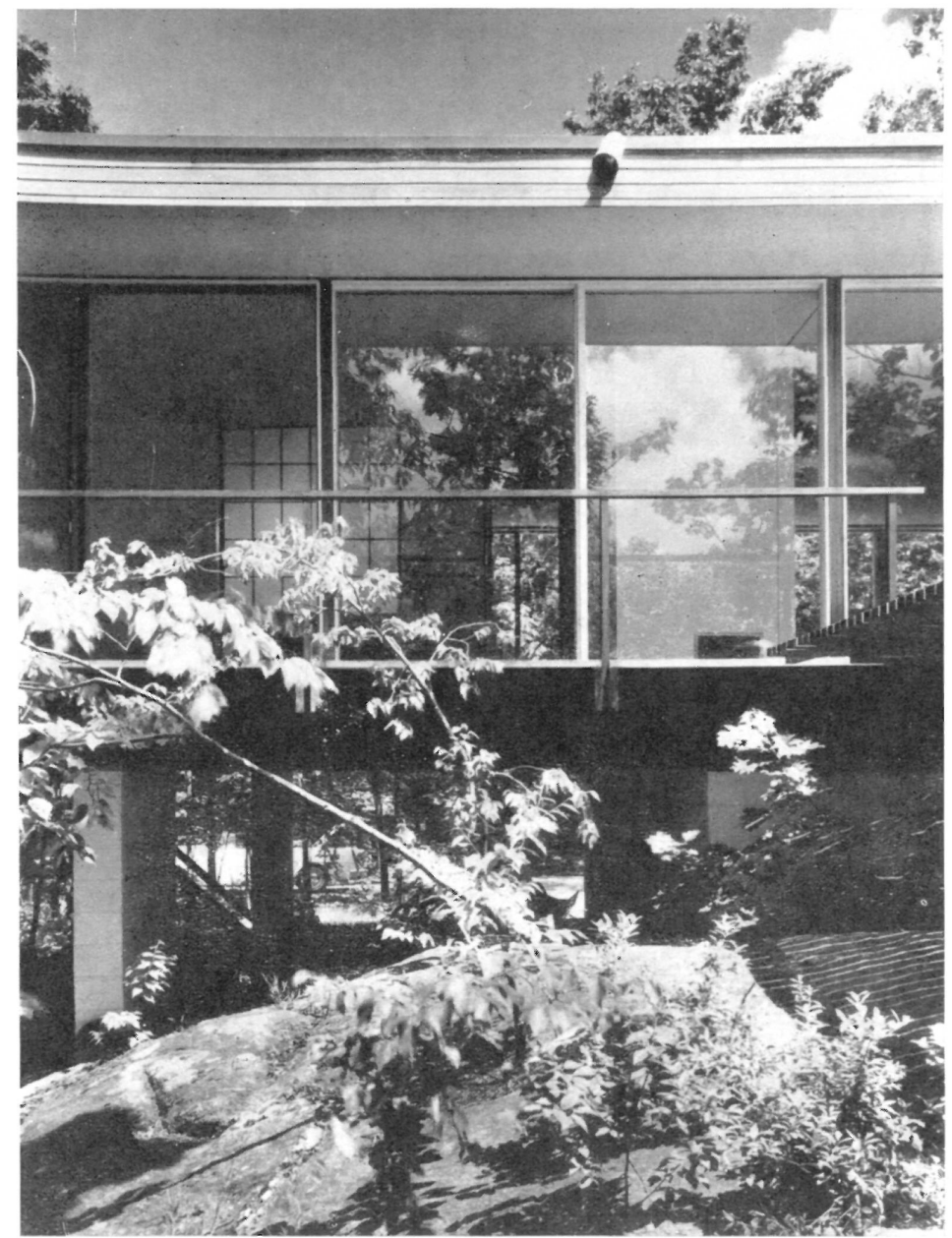




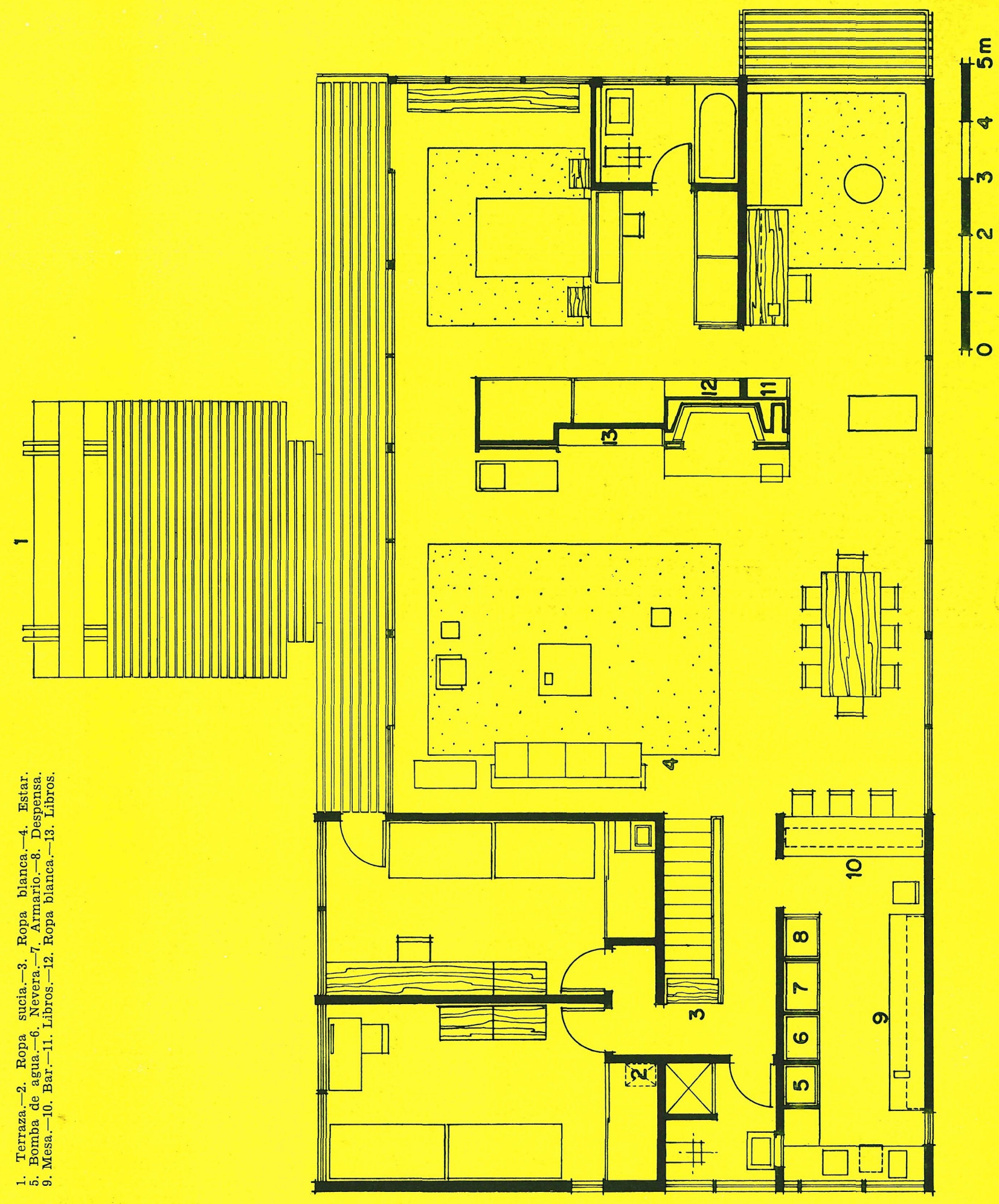

planta primera 


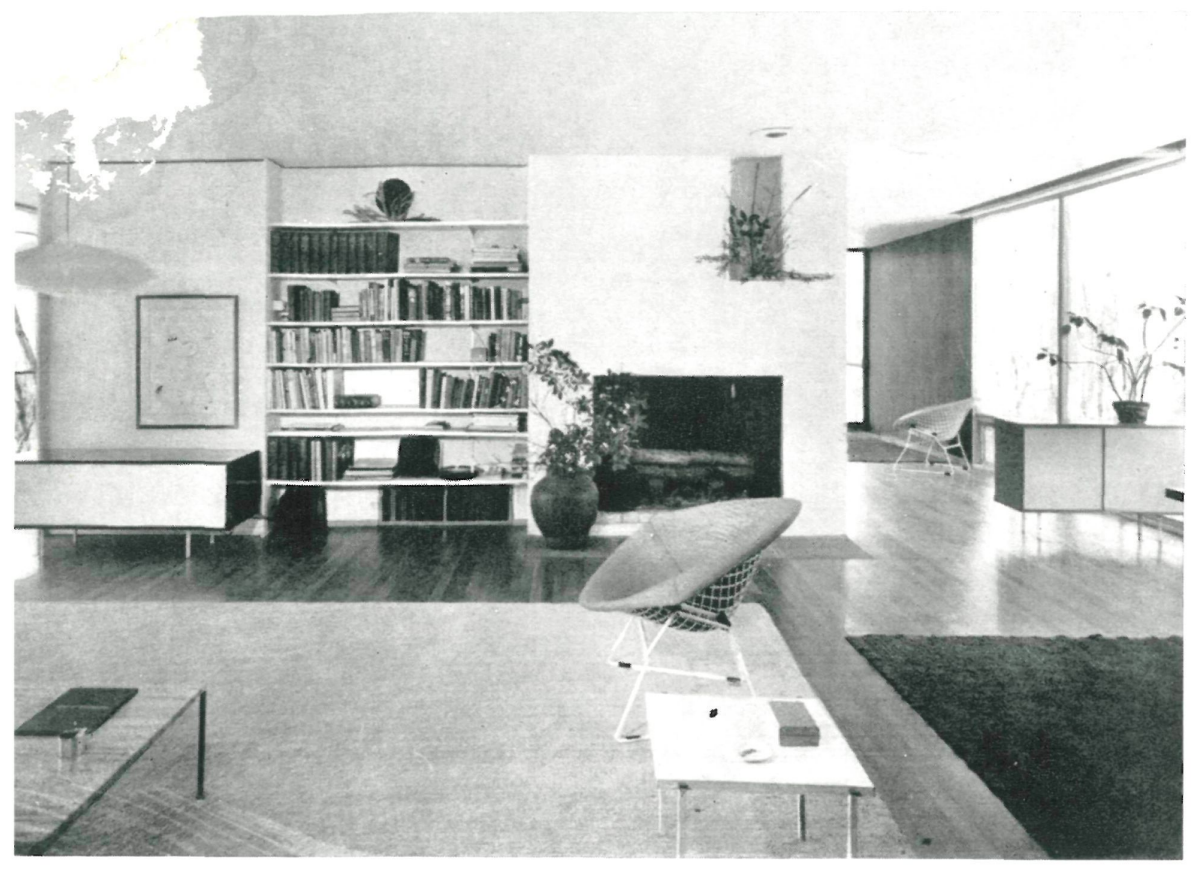

Es de gran interés, en esta vivienda, la distribución de los espacios interiores y su funcionamiento orgánico.

El acceso a la casa conduce directamente al garaje.

La entrada se encuentra en la parte lateral; del vestíbulo se sube, a través de una escalera abierta, a la sala de estar, y con ello al centro de la vivienda.

El comedor y la sala de estar forman un espacio único, en el que una chimenea-hogar, dispuesta en la parte que une el salón con el comedor, ayuda a crear una atmósfera de intimidad.

La separación entre la sala de estar y el dormitorio principal la constituyen, esencialmente, una serie de armarios empotrados; existe, sin embargo, la posibilidad de que dichas habitaciones puedan unirse o separarse mediante una puerta corredera.
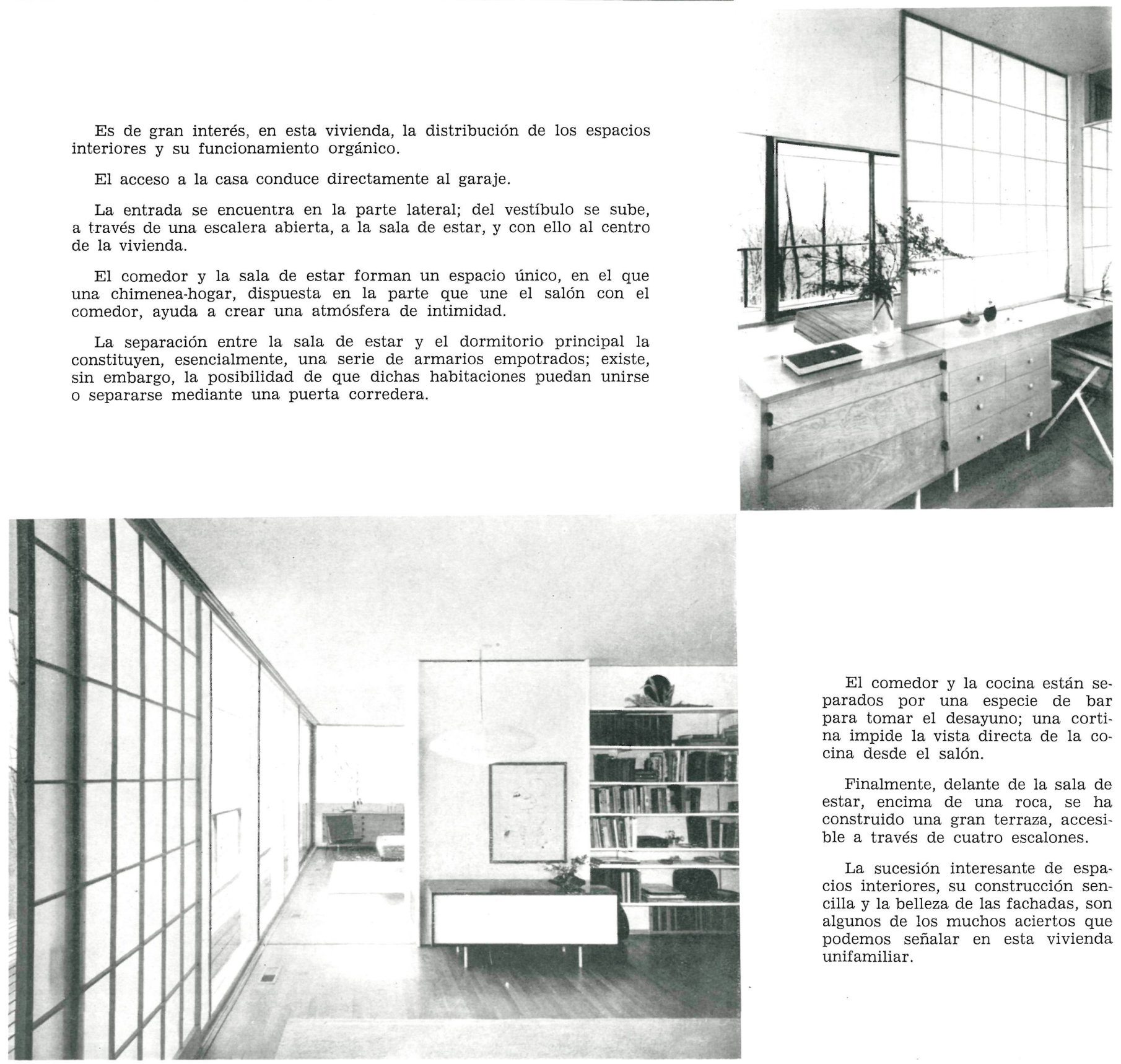

El comedor y la cocina están separados por una especie de bar para tomar el desayuno; una cortina impide la vista directa de la cocina đesde el salón.

Finalmente, delante de la sala de estar, encima de una roca, se ha construido una gran terraza, accesible a través de cuatro escalones.

La sucesión interesante de espacios interiores, su construcción sencilla y la belleza de las fachadas, son algunos de los muchos aciertos que podemos señalar en esta vivienda unifamiliar. 


\section{Une villa entre les mochens}

Ladislav L. Rado, architecte.

L'édifice comporte deux étages: le rez-de-chaussée comprend l'entrée, le vestibule, le garage et les dépendances secondaires, et l'étage supérieur les pièces de séjour.

La villa, proprement dite, s'élève sur une sorte de socle-formé par le bâtiment du rez-de-chaussée-mais comme cette base d'appui est relativement petite et que le rez-de-chaussée repose sur une série de piliers, on éprouve la sensation que le paysage-à cet endroit, vierge et sauvage-pénètre dans la maison, collaborant ainsi au jeu des volumes.

La succession harmonieuse d'espaces intérieurs, la simplicité de sa construction, et la beauté de ses façades, sont d'autant de succès que nous devons signaler dans cette oeuvre si personnelle de Ladislav $\mathbf{L}$. Rado.

\section{Single family dwellihg amidst mocks}

Ladislav L. Rado, architect.

This house has two storeys. Downstairs there is an entrance, a vestibule, secondary services and a garage. Upstairs are the main rooms of the house.

The essential part of the dwelling rests on a kind of wall enclosing the ground floor, but as the latter is very reduced, it is also supported by a number of pillars. The impression is conveyed that the landscape, which is wild and savage in this region, penetrates into the house itself, emphasizing the interplay of masses.

This very personal design by Rado has a series of harmonically related indoor volumes, is of very simple construction, and exhibits beautiful external perspectives.

\section{Eimfoumilienthaus zwischen Felshlöckem}

Ladislav L. Rado, Architekt.

Das Gebäude ist in zwei Stockwerke eingeteilt; im Parterre befinden sich der Eingang mit Vorhalle, Garage und Hauswirtschaftsraum; das Obergeschoss enthält die eigentlichen Wohnräume.

Der eigentliche Wohnteil ruht auf einer Art Sockel, der vom Parterre gebildet wird; doch da diese Stütze relativ klein ist, muss sich das Obergeschoss noch auf eine Reihe von Säulen stützen, wodurch der Eindruck erweckt wird, als ob die Landschaft in ihrer Ursprünglichkeit in das Haus hineindringt und mit an dem interessanten Spiel von Raum Teil hat. 